\title{
A Hybrid Analysis for Security Protocols with State*
}

\author{
John D. Ramsdell \\ Joshua D. Guttman \\ Daniel J. Dougherty \\ Paul D. Rowe
}

September 10, 2018

\begin{abstract}
Cryptographic protocols rely on message-passing to coordinate activity among principals. Many richly developed tools, based on wellunderstood foundations, are available for the design and analysis of pure message-passing protocols. However, in many protocols, a principal uses non-local, mutable state to coordinate its local sessions. Cross-session state poses difficulties for protocol analysis tools.

We provide a framework for modeling stateful protocols, and a hybrid analysis method. We leverage theorem-proving - specifically, PVS - for reasoning about computations over state. An "enrich-byneed" approach - embodied by CPSA — focuses on the message-passing part. The Envelope Protocol, due to Mark Ryan furnishes a case study.
\end{abstract}

Protocol analysis is largely about message-passing in a model in which every message transmitted is made available to the adversary. The adversary can deliver the messages transmitted by the regular (i.e. compliant) principals, if desired, or not. The adversary can also retain them indefinitely, so that in the future he can deliver them, or messages built from them, repeatedly.

However, some protocols also interact with long-term state. For instance, the Automated Teller Machine protocols interact with the long-term state

${ }^{*}$ This work partially supported by the US National Security Agency, and partially supported by the National Science Foundation under grant CNS-1116557. Authors' email addresses: \{guttman,prowe,ramsdell\}@mitre.org, \{dd,guttman\}@wpi.edu. 
stored in banks' account databases. Protocol actions are constrained by that long-term state; for instance, an ATM machine will be told not to dispense cash to a customer whose account has insufficient funds. Protocol actions cause updates to long-term state; for instance, a successful withdrawal reduces the funds in the customer's account. State-manipulating protocols are important to electronic finance and commerce. They are also important in trusted computing, i.e. in systems using Trusted Platform Modules for attestation and secrecy. Indeed, as software interacts with real-world resources in interoperable ways, cryptographic protocols that manipulate long-term state will be increasingly central.

Long-term state is fundamentally different from message passing. The adversary can always choose to redeliver an old message. But he cannot choose to redeliver an old state; for instance, the adversary in an ATM network cannot choose to replay a withdrawal, applying it to a state in which he has sufficient funds, in case he no longer does. Regular principals maintain long-term state across protocol executions in order to constrain subsequent executions, and ensure that future runs will behave differently from past runs.

The Cryptographic Protocol Shapes Analyzer [24] (CPSA) is our program for automatically characterizing the possible executions of a protocol compatible with a specified partial execution. It is grounded in strand space theory. There exists a mathematically rigorous theory [18] that backs up the implementation of CPSA in Haskell, and proves the algorithm produces characterizations that are complete, and that the algorithm enumerates these characterizations.

Part of state manipulation can be encoded by message-passing. In this "state-passing style," reception of a message bearing the state represents reading from the state, and transmission of an updated state as a message represents writing to the state. These conventions help CPSA analyze protocols with state. If a protocol interacts with the state, we add state-bearing receive/transmit event pairs to its roles, and CPSA attempts to find paths through state space as it generates executions. However, CPSA constructs some executions which are in fact not possible. In these executions, a statebearing message is transmitted from one node and then received by two different state-receiving nodes.

CPSA does not recognize that this is not possible in a state-history, and thus provides only an approximate analysis. Showing the correctness of the protocol requires a more refined analysis. 
Our contribution. We apply CPSA to a system that relies on state, coupling CPSA with the Prototype Verification System [21] (PVS) proof assistant.

We specified a version of strand space theory in PVS. On top of this theory, we encoded the result of a CPSA analysis run as a formula in the PVS logic. This formula is justified by the CPSA completeness result [23]. We then use this formula as an axiom in PVS. Proofs using this axiom may imply the existence of additional message transmission/receptions, leading to an enriched CPSA analysis. In this way the theorem-proving and executionfinding analysis activities cooperate, over the common semantic foundation of strand space theory. Hence, the combination is semantically sound.

Outline of the Analysis. Our paradigm is CPSA's enrich-by-need approach [15]. That is, we ask: What kinds of executions are possible, assuming that a particular pattern of events has occurred? To verify authentication properties, we observe that all executions contain certain required events. To verify confidentiality properties, we consider patterns that include a disclosure, and observing that no executions are possible. Our method involves a conversation (so to speak) between CPSA and PVS. The main steps are:

1. Within PVS we define theories (i) $T_{b n d}$ of strand spaces and protocol executions ("bundles") and (ii) $T_{\text {state }}$ of transition relations and their state histories (see Fig. 1). $T_{\text {annot }}$ is their union, a theory of protocol executions where some protocol steps are annotated with a state transition. Augmenting $T_{b n d}$ with information about a protocol $\Pi$ produces $T_{\text {bnd }}(\Pi)$. Augmenting $T_{\text {state }}$ with information about a particular transition relation $\rightsquigarrow$ produces $T_{\text {state }}(\rightsquigarrow)$. The union of $T_{\text {annot }}, T_{\text {bnd }}(\Pi)$, and $T_{\text {state }}(\rightsquigarrow)$ is $T_{\text {annot }}(\Pi, \rightsquigarrow)$.

Our Pvs theories are in fact somewhat coarser than this.

2. Within the state transition theory $T_{\text {state }}(\rightsquigarrow)$, we prove lemmas in PVS such as Lemma 1 below. Some of their consequences in the annotated protocol theory $T_{\text {annot }}(\Pi, \rightsquigarrow)$ use only the limited vocabulary of $T_{b n d}(\Pi)$; we call them bridge lemmas. Lemma 3 is a bridge lemma. They bring information back from the state world to the protocol world.

3. Independently, CPSA analyzes the protocols, with state-manipulation modeled as message-passing, but without any special knowledge about state transition histories. A sentence, called a shape analysis sentence $[22,15]$, summarizes its results in a sentence in the language 


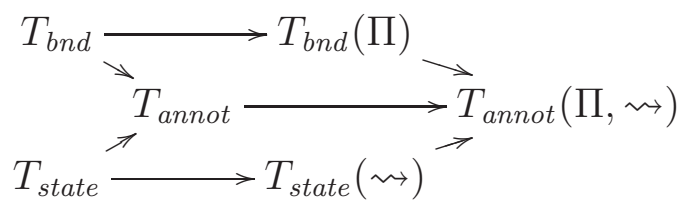

Figure 1: Theory Inclusions

of $T_{b n d}(\Pi)$. A shape analysis sentence, such as Lemma 2, is used as an axiom in proofs within PVs.

4. Using bridge lemmas and state analysis sentences jointly, we infer conclusions about protocol runs in $T_{b n d}(\Pi)$. If we prove a contradiction, that shows that the situation given to CPSA cannot in fact occur. Otherwise, we may prove that additional message transmissions and receptions occurred, as in Thm. 4.

5. We incorporate these additional nodes into a new CPSA starting point, and allow CPSA to draw conclusions. Additional round trips are possible.

Structure. The body of this paper describes an application of our method to the Envelope Protocol, a protocol that interacts with a Trusted Platform Module (TPM) to achieve an important security goal. Section 1 describes the protocol П. Section 2 describes our TPM model, $T_{\text {state }}(\rightsquigarrow)$. Section 3 presents the theory of bundles $T_{b n d}$ encoded within PVS, and specializes this to $T_{b n d}(\Pi)$, demonstrating our main trick of including state-bearing receivetransmit pairs to encode the state transitions. Section 4 describes CPSA, our protocol analysis tool and what results CPSA infers in $T_{b n d}(\Pi)$. Section 5 links the state world and the protocol world $T_{\text {annot }}(\Pi, \rightsquigarrow)$. The relevant bridge lemma is stated and applied to prove the Envelope Protocol security goal.

\section{The Envelope Protocol}

The proof of an important security goal of the Envelope Protocol [2] was the focus of most of our effort. The protocol allows someone to package a secret such that another party can either reveal the secret or prove the secret never was and never will be revealed. 
Protocol motivation. The plight of a teenager motivates the protocol. The teenager is going out for the night, and her parents want to know her destination in case of emergency. Chafing at the loss of privacy, she agrees to the following protocol. Before leaving for the night, she writes her destination on a piece of paper and seals the note in an envelope. Upon her return, the parents can prove the secret was never revealed by returning the envelope unopened. Alternatively, they can open the envelope to learn her destination.

The parents would like to learn their daughter's destination while still pretending that they have respected her privacy. The parents are thus the adversary. The goal of the protocol is to prevent this deception.

Necessity of long-term state. The long-term state is the envelope. Once the envelope is torn open, the adversary no longer has access to a state in which the envelope is intact. A protocol based only on message passing is insufficient, because the ability of the adversary monotonically increases. At the beginning of the protocol the adversary can either return the envelope or tear it. In a purely message-based protocol the adversary will never lose these abilities.

Cryptographic version. The cryptographic version of this protocol uses a TPM to achieve the security goal. Here we restrict our attention to a subset of the TPM's functionality. In particular we model the TPM as having a state consisting of a single Platform Configuration Register (PCR) and only responding to five commands. A boot command sets the PCR to a known value. The extend command takes a piece of data, $d$, and replaces the current value val of the PCR with the hash of $d$ and val, i.e. \# $(d, v a l)$. In fact, the form of extend that we model, which is an extend within an encrypted session, also protects against replay. These are the only commands that alter the value in a PCR.

The TPM provides other services that do not alter the PCR. The quote command reports the value contained in the PCR and is signed in a way as to ensure its authenticity. The create key command causes the TPM can create an asymmetric key pair where the private part remains shielded within the TPM. However, it can only be used for decryption when the PCR has a specific value. The decrypt command causes the TPM to decrypt a message using this shielded private key, but only if the value in the PCR matches the constraint of the decryption key.

In what follows, Alice plays the role of the teenaged daughter packaging the secret. Alice calls the extend command with a fresh nonce $n$ in an 
encrypted session. She uses the create key command constraining that new key to be used only when a specific value is present in the PCR. In particular, the constraining value $c v$ she chooses is the following:

$$
c v=\#(\text { "obtain", \#(n, val) })
$$

where val was the PCR value prior the extend command. She then encrypts her secret $v$ with this newly created key.

Using typical message passing notation, Alice's part of the protocol might be represented as follows (where $k^{\prime}$ denotes the key created in the second line, and where we still ignore the replay protection):

$$
\begin{aligned}
\mathrm{A} & \rightarrow \mathrm{TPM}:\{\mid \text { "extend", } n \mid\}_{k} \\
\mathrm{~A} & \rightarrow \mathrm{TPM}: \text { "create key", \#("obtain", \#(n,val)) } \\
\mathrm{TPM} & \rightarrow \mathrm{A}: k^{\prime} \\
\mathrm{A} & \rightarrow \text { Parent }:\{|v|\}_{k^{\prime}}
\end{aligned}
$$

The parent acts as the adversary in this protocol. We assume he can perform all the normal Dolev-Yao operations such as encrypting and decrypting messages when he has the relevant key, and interacting with honest protocol participants. Most importantly, the parent can use the TPM commands available in any order with any inputs he likes. Thus he can extend the PCR with the string obtain and use the key to decrypt the secret. Alternatively, he can extend the PCR with the string refuse and then generate a TPM quote as evidence the secret will never be exposed. The goal of the Envelope Protocol is to ensure that once Alice has prepared the TPM and encrypted her secret, the parent should not be able to both decrypt the secret and also generate a refusal quote, $\left\{\mid \text { "quote", \#("refuse", \#(n,val)), }\{|v|\}_{k^{\prime}} \mid\right\}_{\text {aik }}$.

A crucial fact about the PCR role in this protocol is the injective nature of the hashing, ensuring that for every $x$

$$
\text { \#("obtain", \#(n,val)) } \neq \quad \text { \#("refuse", } x)
$$

\section{The TPM Model}

In this section we introduce our TPM state theory $T_{\text {state }}(\rightsquigarrow)$ focusing on representing the value of the PCR and how the TPM commands may change it. 


\begin{tabular}{|c|c|c|}
\hline Sorts: & $\mathrm{M}, T, \mathrm{~A}, \mathrm{~S}, \mathrm{D}, \mathrm{E}$ & \\
\hline Subsorts: & $\mathrm{A}<\mathrm{T}, \mathrm{S}<\mathrm{T}, \mathrm{D}<\mathrm{T}$ &, $\mathrm{E}<\top$ \\
\hline Operations: & $: M$ & TPM boot \\
\hline & $: \top \times M \rightarrow M$ & TPM extend \\
\hline & $: \top \times \top \rightarrow \top$ & Pairing \\
\hline & $\{|\cdot|\}_{(\cdot)}: \top \times \mathrm{A} \rightarrow \top$ & Asymmetric encryption \\
\hline & $\{|\cdot|\}_{(\cdot)}: \top \times S \rightarrow \top$ & Symmetric encryption \\
\hline & $(\cdot)^{-1}: A \rightarrow A$ & Asymmetric key inverse \\
\hline & $(\cdot)^{-1}: S \rightarrow S$ & Symmetric key inverse \\
\hline & $: \top \rightarrow S$ & Hashing \\
\hline & $\mathrm{a}_{i}, \mathrm{~b}_{i}: \mathrm{A}$ & Asymmetric key constants \\
\hline & $: S$ & Symmetric key constants \\
\hline & : D & Data constants \\
\hline & : $E$ & Text constants \\
\hline Equations: & $\begin{array}{l}\mathrm{g}_{i} \quad: \top \\
\mathrm{a}_{i}{ }^{-1}=\mathrm{b}_{i} \quad \mathrm{~b}_{i}{ }^{-1}=\mathrm{a}_{i} \\
\forall k: \text { A. }\left(k^{-1}\right)^{-1}=k\end{array}$ & $\begin{array}{l}\text { Tag constants } \\
(i \in \mathbb{N}) \\
\forall k: \text { S. } k^{-1}=k\end{array}$ \\
\hline
\end{tabular}

Figure 2: Crypto Algebra with State Signature

Fig. 2 shows the signature of the order-sorted algebra used in this paper. Sort $M$ is the sort of TPM machine states and sort $T$ is the top sort of messages. Messages of sort A (asymmetric keys), sort S (symmetric keys), sort D (data), and sort E (text) are called atoms. Messages are atoms, tag constants, or constructed using encryption $\{|\cdot|\}_{(\cdot)}$, hashing \#(.), and pairing $(\cdot, \cdot)$, where the comma operation is right associative and parentheses are omitted when the context permits.

The algebra is the initial quotient term algebra over the signature. It is easy to show that each term $t$ of the algebra is equal to a unique term $t^{\prime}$ with no occurrences of the inverse operation $(\cdot)^{-1}$; we choose this $t^{\prime}$ to be the canonical representative of $t$.

We use the function pcr to coerce TPM states, which are of sort M, to messages, specifically to symmetric keys of sort S:

$$
\begin{gathered}
p c r: \mathrm{M} \rightarrow \mathrm{S} \\
\operatorname{pcr}(\mathrm{bt})=\mathrm{s}_{0} \\
\operatorname{pcr}(\operatorname{ex}(t, m))=\#(t, p c r(m))
\end{gathered}
$$


where constant $\mathrm{s}_{0}$ is known to all. Modeling the injectivity of the hash function (cf. Equation 1), we postulate that the function pcr is injective.

The definition of the TPM transition relation $\rightsquigarrow$ is

$$
\begin{array}{rrrr}
m_{0} \rightsquigarrow m_{1} & \text { iff } & m_{1}=\text { bt } & \text { (boot) } \\
\text { or } & \exists t: \top \cdot m_{1}=\operatorname{ex}\left(t, m_{0}\right) & \text { (extend) } \\
\text { or } & m_{0}=m_{1} & \text { (quote, decrypt) }
\end{array}
$$

The create key command does not interact with the state.

In this framework we prove a crucial property of all executions which we express in terms of the notion of a state having a message. A state has a message if an extend operation with it is part of the state. For example, ex("obtain", ex $(v, \mathrm{bt}))$ has "obtain" and $v$, but it does not have "refuse".

An infinite sequence of states $\pi$ is a path if $\pi(0)=$ bt and $\forall i \in \mathbb{N}$. $(\pi(i)$, $\pi(i+1)) \in \rightsquigarrow$. Paths in this TPM model have several useful properties. For example, if a previous state is not a subterm of a state, there must have been an intervening boot. Also, if a state has a message, and a previous state is a boot state, there must have been an intervening transition that extends with the message. These two properties can be combined into the property used by the proof of the Envelope Protocol security goal: if a previous state is not

a subterm of a state that has a message, there must have been an intervening transition that extends with the message. Lemma 1 formalizes this property in our state theory $T_{\text {state }}(\rightsquigarrow)$, and we proved it using PVS.

Lemma 1 (Prefix Boot Extend).

$$
\begin{aligned}
& \forall \pi \in \text { path }, t: \top, i, k \in \mathbb{N} . i \leq k \wedge \pi(k) \text { has } t \\
& \supset \operatorname{subterm}(\pi(i), \pi(k)) \\
& \quad \vee \exists j \in \mathbb{N} . i \leq j<k \wedge \pi(j+1)=\operatorname{ex}(t, \pi(j))
\end{aligned}
$$

\section{Strand Spaces}

This section introduces our strand space theory of the envelope protocol, $T_{\text {bnd }}(\Pi)$. In strand space theory [25], a strand represents the local behavior of a principal in a single session. The trace of a strand is a linearly ordered sequence of events $e_{0} \Rightarrow \cdots \Rightarrow e_{n-1}$, and an event is either a message transmission $+t$ or a reception $-t$, where $t$ has sort $T$. A strand space $\Theta$ is a map from a set of strands to a set of traces. In the PVs theory of strand spaces, 
the set of strands is a prefix of the natural numbers, so a strand space is a finite sequence of traces.

In a strand space, a node identifies an event. The nodes of strand space $\Theta$ are $\{(s, i)|s \in \operatorname{Dom}(\Theta), 0 \leq i<| \Theta(s) \mid\}$, and the event at a node is $\operatorname{evt}_{\Theta}(s, i)=\Theta(s)(i)$.

A message $t_{0}$ is carried by $t_{1}$, written $t_{0} \sqsubseteq t_{1}$ if $t_{0}$ can be extracted from a reception of $t_{1}$, assuming the necessary keys are available. In other words, $\sqsubseteq$ is the smallest reflexive, transitive relation such that $t_{0} \sqsubseteq t_{0}, t_{0} \sqsubseteq\left(t_{0}, t_{1}\right)$, $t_{1} \sqsubseteq\left(t_{0}, t_{1}\right)$, and $t_{0} \sqsubseteq\left\{\left|t_{0}\right|\right\}_{t_{1}}$. A message originates in trace $c$ at index $i$ if it is carried by $c(i), c(i)$ is a transmission, and it is not carried by any event earlier in the trace. A message $t$ is non-originating in a strand space $\Theta$, written non $(\Theta, t)$, if it originates on no strand. A message $t$ uniquely originates in a strand space $\Theta$ at node $n$, written $\operatorname{uniq}(\Theta, t, n)$, if it originates in the trace of exactly one strand $s$ at index $i$, and $n=(s, i)$.

The model of execution is a bundle. The pair $\Upsilon=(\Theta, \rightarrow)$ is a bundle if it defines a finite directed acyclic graph, where the vertices are the nodes of $\Theta$, and an edge represents communication $(\rightarrow)$ or strand succession $(\Rightarrow)$ in $\Theta$. For communication, if $n_{0} \rightarrow n_{1}$, then there is a message $t$ such that $e v t_{\Theta}\left(n_{0}\right)=+t$ and $\operatorname{evt}_{\Theta}\left(n_{1}\right)=-t$. For each reception node $n_{1}$, there is a unique transmission node $n_{0}$ with $n_{0} \rightarrow n_{1}$. We use $\prec$ to denote the causal ordering of nodes in a bundle: the transitive closure of $\rightarrow \cup \Rightarrow$. The strand space associated with a bundle $\Upsilon$ will be denoted $\Theta_{\Upsilon}$ unless the association is clear from the context.

When a bundle is a run of a protocol, the behavior of each strand is constrained by a role. Adversarial strands are constrained by roles as are non-adversarial strands. A protocol is a set of roles, and a role is a set of traces. A trace $c$ is an instance of role $r$ if $c$ is a prefix of some member of $r$. More precisely, for protocol $P$, we say that bundle $\Upsilon=(\Theta, \rightarrow)$ is a run of protocol $P$ if there exists a role assignment $r a \in \operatorname{Dom}(\Theta) \rightarrow P$ such that for all $s \in \operatorname{Dom}(\Theta), \Theta(s)$ is an instance of $r a(s)$. In what follows, we fix the protocol $P$ and only consider bundles that are runs of $P$.

The roles that constrain adversarial behavior are defined by the functions in Figure 3. The adversary can execute all instances of these patterns. For the encryption related roles, $k: \mathrm{A} \mid \mathrm{S}$ asserts that $k$ is either a symmetric or asymmetric key. For the create role, $t: A|S| D \mid E$ asserts that $t$ is an atom. Atoms, characteristically, are what the adversary can create out of thin air (modulo origination assumptions).

There is a role for each TPM operation. We represent them using a state- 


$$
\begin{aligned}
\operatorname{create}(t: \mathrm{A}|\mathrm{S}| \mathrm{D} \mid \mathrm{E}) & =+t \quad t a g_{i}=+g_{i} \\
\operatorname{pair}\left(t_{0}: \top, t_{1}: \top\right) & =-t_{0} \Rightarrow-t_{1} \Rightarrow+\left(t_{0}, t_{1}\right) \\
\operatorname{sep}\left(t_{0}: \top, t_{1}: \top\right) & =-\left(t_{0}, t_{1}\right) \Rightarrow+t_{0} \Rightarrow+t_{1} \\
\operatorname{enc}(t: \top, k: \mathrm{A} \mid \mathrm{S}) & =-t \Rightarrow-k \Rightarrow+\{|t|\}_{k} \\
\operatorname{dec}(t: \top, k: \mathrm{A} \mid \mathrm{S}) & =-\{|t|\}_{k} \Rightarrow-k^{-1} \Rightarrow+t \\
\operatorname{hash}(t: \top) & =-t \Rightarrow+\# t
\end{aligned}
$$

Figure 3: Adversary Traces

passing style. The state-passing style allows CPSA to do draw conclusions about where states could come from. Each role receives a message encoding the state at the time it occurs. It transmits a message encoding the state after any state change it causes. We do the encoding using a special tag $\mathrm{g}_{0}$ and an encryption. For a transition $m_{0} \rightsquigarrow m_{1}$, the role contains

$$
\cdots \Rightarrow-\left\{\left|g_{0}, \operatorname{pcr}\left(m_{0}\right)\right|\right\}_{\# k} \Rightarrow+\left\{\left|g_{0}, \operatorname{pcr}\left(m_{1}\right)\right|\right\}_{\# k} \Rightarrow \cdots \text {. }
$$

Here $k$ is an uncompromised symmetric key used only in TPM operations. The states are encoded as encryptions using the hash $\# k$ of $k$. Tag $\mathrm{g}_{0}$ is included to ensure that a state-bearing message is never confused with any other protocol message. State-passing style is less restrictive than actual state histories, since a state-bearing message may be received many times, even if it is sent only once.

Using these receive-transmit pairs of state-bearing messages the TPM roles are represented in Fig. 4, where tag $g_{1}$ is obtain and tag $g_{2}$ is refuse. In the extend role, we now show the two initial messages that provide replay prevention; the TPM supplies a fresh nonce as a session ID that must appear with the value to be extended into the PCR. The createkey role does not interact with the state. It simply creates a key that will be constrained by the state in the boot role.

Alice's role, including the messages to prevent replays, is:

$$
\begin{aligned}
& \text { alice }(\text { sid, } v: \mathrm{D}, \text { esk : } \mathrm{S}, k, \text { tpmk, aik }: \mathrm{A}, n: \mathrm{E}, p: \mathrm{\top})= \\
& +\left(\mathrm{g}_{4}, \text { tpmk, }\{\mid \text { esk } \mid\}_{\text {tpmk }}\right) \Rightarrow-\left(\mathrm{g}_{4}, \text { sid }\right) \\
& \quad \Rightarrow+\left\{\mid \mathrm{g}_{5}, n, \text { sid } \mid\right\}_{\text {esk }} \Rightarrow+\left(\mathrm{g}_{9}, \#\left(\mathrm{~g}_{1}, \#(n, p)\right)\right) \\
& \quad \Rightarrow-\left\{\left|\mathrm{g}_{8}, \#\left(\mathrm{~g}_{1}, \#(n, p)\right)\right|\right\}_{\text {aik }} \Rightarrow+\{|v|\}_{k}
\end{aligned}
$$

The parameters sid and tpmk help prevent replays. To make formulas more comprehensible, we omit them. 


$$
\begin{aligned}
& \operatorname{boot}(k: \mathrm{S}, p: \top)= \\
& -\mathrm{g}_{3} \Rightarrow-\left\{\left|\mathrm{g}_{0}, p\right|\right\}_{\# k} \Rightarrow+\left\{\left|\mathrm{g}_{0}, \mathrm{~s}_{0}\right|\right\}_{\# k} \\
& \text { extend(sid: D, tpmk: } \mathrm{A}, e s k, k: \mathrm{S}, p, t: \top)= \\
& -\left(\mathrm{g}_{4}, t p m k,\{|e s k|\}_{\text {tpmk }}\right) \Rightarrow+\left(\mathrm{g}_{4}, \text { sid }\right) \Rightarrow-\left\{\mid \mathrm{g}_{5}, t, \text { sid } \mid\right\}_{\text {esk }} \\
& \Rightarrow-\left\{\left|\mathrm{g}_{0}, p\right|\right\}_{\# k} \Rightarrow+\left\{\left|\mathrm{g}_{0}, \#(t, p)\right|\right\}_{\# k} \\
& \text { quote }(k: \mathrm{S}, \text { aik }: \mathrm{A}, p, n: \top)= \\
& -\left(\mathrm{g}_{6}, n\right) \Rightarrow-\left\{\left|\mathrm{g}_{0}, p\right|\right\}_{\# k} \Rightarrow+\left\{\left|\mathrm{g}_{0}, p\right|\right\}_{\# k} \Rightarrow+\left\{\left|\mathrm{g}_{6}, p, n\right|\right\}_{\text {aik }} \\
& \operatorname{decrypt}\left(m, t: \top, k^{\prime}, \text { aik }: \mathrm{A}, k: \mathrm{S}\right)= \\
& -\left(\mathrm{g}_{7},\{|m|\}_{k^{\prime}}\right) \Rightarrow-\left\{\left|\mathrm{g}_{8}, k^{\prime}, p\right|\right\}_{a i k} \Rightarrow-\left\{\left|\mathrm{g}_{0}, p\right|\right\}_{\# k} \Rightarrow+\left\{\left|\mathrm{g}_{0}, p\right|\right\}_{\# k} \Rightarrow+m \\
& \text { createkey }(k, \text { aik }: \mathrm{A}, t: \top)= \\
& -\left(\mathrm{g}_{9}, t\right) \Rightarrow+\left\{\left|\mathrm{g}_{8}, k, t\right|\right\}_{\text {aik }} \\
& \mathrm{g}_{0} \text { state } \mathrm{g}_{2} \text { refuse } \mathrm{g}_{4} \text { session } \mathrm{g}_{6} \text { quote } \mathrm{g}_{8} \text { created } \\
& g_{1} \text { obtain } g_{3} \text { boot } g_{5} \text { extend } g_{7} \text { decrypt } g_{9} \quad \text { create key }
\end{aligned}
$$

Figure 4: State-Bearing Traces

\section{CPSA}

This section discusses how we use our analysis tool CPSA to infer results in the theory $T_{b n d}(\Pi)$. CPSA carries out enrich-by-need analysis, and characterizes the set of bundles consistent with a partial description of a bundle.

These partial descriptions are called skeletons. CPSA takes as input an initial skeleton $\mathbb{A}_{0}$, and when it terminates it outputs a set of more descriptive skeletons $\left\{\mathbb{B}_{i}\right\}_{i \in I}$. They have the property that any bundle containing the structure in the initial skeleton $\mathbb{A}_{0}$ also contains all the structure in one of the output skeletons $\mathbb{B}_{i}$. In particular, it infers all of the non-adversarial behavior that must be present in any bundle satisfying the initial description. Of course for some initial skeletons $\mathbb{A}_{0}$, there may be no bundles that are consistent with them. In this case, CPSA outputs the empty set.

The security goal for the Envelope Protocol is that a run of Alice's role should ensure that the secret and the refusal certificate are not both available:

Security Goal 1. Consider the following events: 


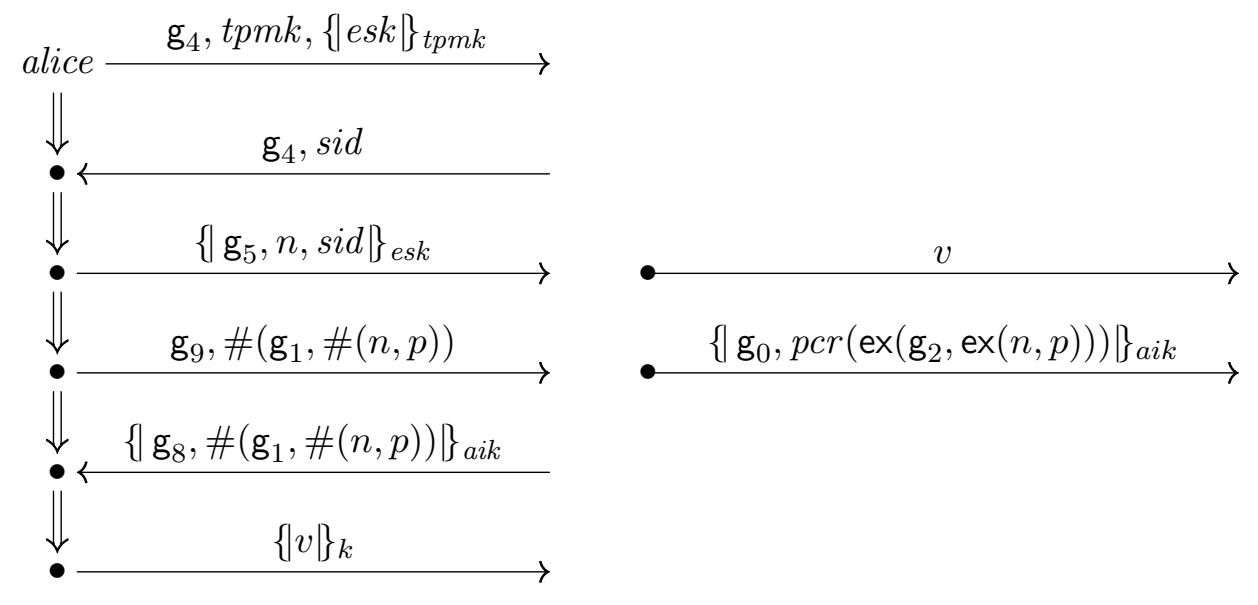

Figure 5: Alice Point-Of-View

- An instance of the Alice role runs to completion, with secret $v$ and nonce $n$ both freshly chosen;

- $v$ is observed unencrypted;

- the refusal certificate $\left.\{\mid \text { "quote", \#("refuse", \#(n,val)), \{|v|\}}\}_{k^{\prime}} \mid\right\}_{\text {aik }}$ is observed unencrypted.

These events, which we call jointly $\mathbb{A}_{0}$, are not all present in any execution.

We can feed CPSA an input skeleton $\mathbb{A}_{0}$ representing this undesirable situation. The skeleton $\mathbb{A}_{0}$ is visualized in Fig. 5 .

We would hope CPSA could determine that no bundles are consistent with this input $\mathbb{A}_{0}$ and return the empty set. However, our technique of using state-bearing messages to represent the TPM state transitions underconstrains the set of possible state paths. For this reason, CPSA actually produces one skeleton in its output. This skeleton represents some activity that must have occured within the TPM in any bundle conforming to the initial skeleton. It contains an instance of the decrypt role (to explain the secret leaking), an instance of the quote role (to explain the creation of the refusal token), and several instances of the extend role (to explain how the TPM state evolved in order to allow the other two operations).

Fig. 6 displays the relevant portion of CPSA's output displaying only the state-bearing nodes of the extend strands inferred by CPSA. Notice that two 


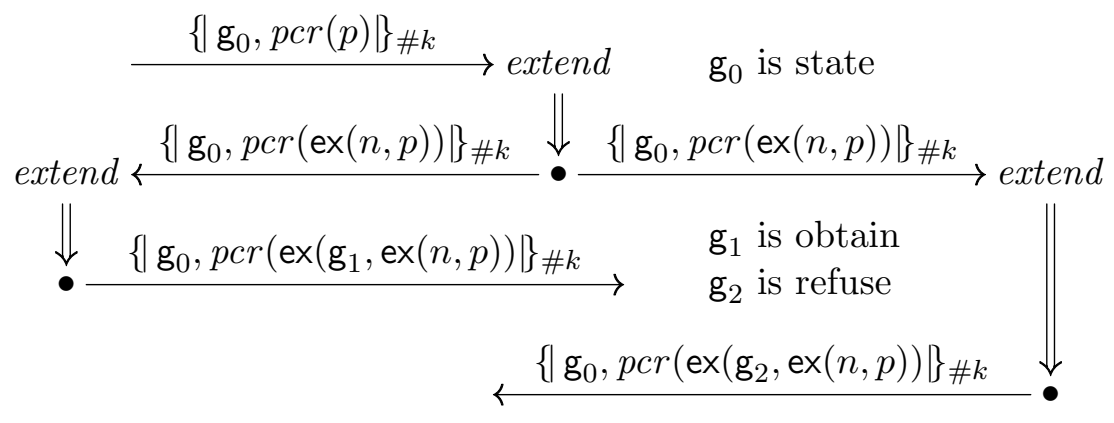

Figure 6: State Splitting

of the extend strands branch off from the third strand. This is a state split in which a single state evolves in two distinct ways. The technique of using state-bearing messages is not sufficient to preclude this possibility.

CPSA's enrich-by-need approach is a form of model finding, rather than theorem proving. In order to use CPSA's results to our advantage we need to express its conclusions in the logical theory $T_{b n d}(\Pi)$. For that purpose we transform our skeletons into formulas in order-sorted logic and define what it means for a bundle to satisfy these formulas. The sorts are the message algebra sorts augmented with a sort $\mathbf{Z}$ for strands and sort $\mathrm{N}$ for nodes. The atomic formula htin $(z, h, c)$ asserts that strand $z$ has a length of at least $h$, and its trace is a prefix of trace $c$. The formula $n_{0} \ll n_{1}$ asserts node $n_{0}$ precedes node $n_{1}$. The formula non $(t)$ asserts that message $t$ is non-originating, and uniq $(t, n)$ asserts that message $t$ uniquely originates at node $n$. Finally, the formula sends $(n, t)$ asserts that the event at node $n$ is a transmission of message $t$. The roles of the protocol serve as function symbols. A skeleton $\mathbb{A}$ is represented by the conjunction of all facts true in the skeleton.

We encode an entire CPSA analysis by first encoding the input skeleton $\mathbb{A}_{0}$ and the output skeletons $\left\{\mathbb{B}_{i}\right\}_{i \in I}$. The analysis is then encoded as an implication. A formula $\Phi_{0}$ describing the input $\mathbb{A}_{0}$, is the hypothesis of the conditional. The disjunction of the formulas $\Psi_{i}$ describing the outputs $\left\{\mathbb{B}_{i}\right\}_{i \in I}$ form the conclusion. When CPSA discovers that there are no bundles compatible with the initial skeleton, the conclusion is encoded as the empty disjunction, $\perp$.

The satisfaction relation is defined using the clauses in Fig. 7. It relates a bundle, a variable assignment, and a formula: $\Upsilon, \alpha \models \Phi$. A bundle $\Upsilon$ 


$$
\begin{array}{lc}
\Upsilon, \alpha \models x=y & \text { iff } \alpha(x)=\alpha(y) ; \\
\Upsilon, \alpha \models \operatorname{htin}(z, h, c) & \text { iff }\left|\Theta_{\Upsilon}(\alpha(z))\right| \geq \alpha(h) \text { and } \\
& \Theta_{\Upsilon}(\alpha(z)) \text { is a prefix of } \alpha(c) ; \\
\Upsilon, \alpha \models n_{0} \ll n_{1} & \text { iff } \alpha\left(n_{0}\right) \prec \Upsilon \alpha\left(n_{1}\right) ; \\
\Upsilon, \alpha \models \operatorname{non}(t) & \text { iff } n o n\left(\Theta_{\Upsilon}, \alpha(t)\right) ; \\
\Upsilon, \alpha \models \operatorname{uniq}(t, n) & \text { iff } u n i q\left(\Theta_{\Upsilon}, \alpha(t), \alpha(n)\right) ; \\
\Upsilon, \alpha \models \operatorname{sends}(n, t) & \text { iff } \operatorname{evt}_{\Theta_{\Upsilon}}(\alpha(n))=+\alpha(t) .
\end{array}
$$

Figure 7: Satisfaction

is described by a skeleton iff the skeleton's sentence $\Phi$ satisfies $\Upsilon$, written $\Upsilon \models \Phi$.

The formula $\Phi_{0}$ that specifies the initial skeleton relevant to the Envelope Protocol security goal is

$$
\begin{aligned}
\operatorname{htin} & (z, 4, \operatorname{alice}(v, e s k, k, \operatorname{aik}, n, p)) \wedge \operatorname{sends}\left(n_{1}, v\right) \\
& \wedge \operatorname{sends}\left(n_{2},\left\{\mid \mathrm{g}_{0}, \operatorname{pcr}\left(\operatorname{ex}\left(\mathrm{g}_{2}, \operatorname{ex}(n, p)\right)\right)\right\}_{\text {aik }}\right) \\
& \wedge \operatorname{non}(\operatorname{aik}) \wedge \operatorname{non}(e s k) \\
& \wedge \operatorname{uniq}(n,(z, 1)) \wedge \operatorname{uniq}(v,(z, 4)),
\end{aligned}
$$

where $v: \mathrm{D}$, esk : S, $k$, aik : A, $n: \mathrm{E}, p: \top, z: \mathrm{Z}, n_{1}, n_{2}: \mathrm{N}$.

The output skeleton $\mathbb{B}_{1}$ is much larger and its formula $\Psi_{1}$ is correspondingly large. The relevant part of this formula representing the fragment in Fig. 6 is

$$
\begin{aligned}
& \operatorname{htin}\left(z_{1}, 3, \operatorname{extend}(\operatorname{esk}, k, p c r(p), n)\right) \\
& \quad \wedge \operatorname{htin}\left(z_{2}, 3 \text {, extend }\left(e s k, k, p c r(\operatorname{ex}(n, p)), \mathrm{g}_{1}\right)\right) \\
& \quad \wedge \operatorname{htin}\left(z_{3}, 3, \operatorname{extend}\left(e s k, k, p c r(\operatorname{ex}(n, p)), \mathrm{g}_{2}\right)\right),
\end{aligned}
$$

where esk, $k: \mathrm{S}, p: \top, n: \mathrm{E}, z_{1}, z_{2}, z_{3}: \mathrm{Z}$. The full formula for $\mathbb{B}_{1}$ has more conjuncts.

Let the vector $\bar{x}$ contain the variables that appear free in $\Phi_{0}$, and possibly also in $\Psi_{1}$, and let the vector $\bar{y}$ contain the variables that occur free in $\Psi_{1}$ only. Summarizing CPSA's analysis for the Envelope Protocol in $T_{b n d}(\Pi)$, we have:

Lemma 2. $\forall \bar{x} .\left(\Phi_{0} \supset \exists \bar{y} . \Psi_{1}\right)$, where $\Phi_{0}, \Psi_{1}$ are as in formulas 2-3.

However, unlike Lemma 1, this lemma was not derived within PVs. Rather, it is true if CPSA's analysis is correct. We import it into PVS as an axiom. 
Lemma 2 is however something capable of direct proof within PVS as a theorem of $T_{b n d}(\Pi)$. Indeed, there is precedent for constructing proofs of this sort. Meier et al. [19] show how to instrument a different protocol analysis tool, called Scyther [7], so that each step it takes generates a lemma in the Isabelle proof system. Then, they use reusable results proved once within Isabelle to discharge these lemmas. Curiously, one of the main lemmas, the authentication test theorem in an earlier form, has already been established within PVS [17]. Thus, it appears possible, although a substantial undertaking, to transform CPSA from a central piece of our analysis infrastructure to a heuristic to guide derivations within PVs.

\section{Reasoning About Messages and State}

This section presents some details of the theory $T_{\text {annot }}(\Pi, \rightsquigarrow)$. We then show how the previous lemmas combine allowing us to conclude that the security goal of the Envelope Protcol is achieved.

In $T_{\text {annot }}(\Pi, \rightsquigarrow)$, the state transitions associated with a protocol are specified by annotating some events in a role of $\Pi$ with a subset of the transition relation $\rightsquigarrow$. The reason for annotating events with a subset of the transition relation, rather than an element, will be explained at the end of this section. We use $\perp$ for an event that is not annotated, and $\uparrow a$ for an event that is annotated with $a$. The events that are annotated are the transmissions associated with receive-transmit pairs of state-bearing messages.

$$
\begin{array}{ccc}
\ldots \Rightarrow-\left\{\left|\mathrm{g}_{0}, \operatorname{pcr}\left(m_{0}\right)\right|\right\}_{\# k} \Rightarrow+ & +\left\{\left|\mathrm{g}_{0}, \operatorname{pcr}\left(m_{1}\right)\right|\right\}_{\# k} \Rightarrow \\
\perp & \uparrow & \uparrow\left\{\left(m_{0}, m_{1}\right)\right\} \cap \rightsquigarrow
\end{array}
$$

A node in a bundle inherits its annotation from its role. The set of nodes in $\Upsilon$ that are annotated is anode $(\Upsilon)$, and anno $(\Upsilon, n, a)$ asserts that node $n$ in $\Upsilon$ is annotated with some $a \subseteq \rightsquigarrow$. In the Envelope Protocol, a node annotated by a TPM extend role cannot be an instance of any other role.

Our goal is to reason only with bundles that respect state semantics. A bundle $\Upsilon$ with a transition annotating role assignment is compatible [14, Def. 11] with transition relation $\rightsquigarrow$ if there exists $\ell \in \mathbb{N}, f \in \operatorname{anode}(\Upsilon) \rightarrow$ $\{0,1, \ldots, \ell-1\}$, and $\pi \in$ path such that

1. $f$ is bijective;

2. $\forall n_{0}, n_{1} \in \operatorname{anode}(\Upsilon) . n_{0} \prec n_{1} \Longleftrightarrow f\left(n_{0}\right)<f\left(n_{1}\right)$; 
3. $\forall n \in \operatorname{anode}(\Upsilon), a \subseteq \rightsquigarrow$.

$$
\operatorname{anno}(\Upsilon, n, a) \supset(\pi(f(n)), \pi(f(n)+1)) \in a .
$$

A bundle that satisfies $T_{\text {annot }}(\Pi, \rightsquigarrow)$ is a compatible bundle.

Because the function $f$ is bijective, all annotated nodes in a compatible bundle are totally ordered. Looking back at Fig. 6, either the nodes in the leftmost strand precede the nodes in the rightmost strand or succeed them.

The compatible bundle assumption allows one to infer the existence of nodes that are not revealed by CPSA. In the case of the Envelope Protocol this is done by importing the Prefix Boot Extend Lemma (Lemma 1) from $T_{\text {state }}(\rightsquigarrow)$ into the strand space world by proving the following lemma (stated here in plain English) within $T_{\text {annot }}(\Pi, \rightsquigarrow)$ using PVs. Its proof uses the full content of compatibility.

Lemma 3 (Bridge, informally). Let $\Upsilon$ be a compatible bundle, containing two annotated nodes, $n_{0} \prec n_{1}$, where $n_{1}$ 's state has a value $t$. Then either $n_{0}$ 's state is a subterm of $n_{1}$ 's state, or else there is an extend node between them that incorporates $t$.

This Bridge Lemma implies there is another extend strand between the two strands that represent the state split. This theorem is also proved with PVS in $T_{\text {annot }}(\Pi, \rightsquigarrow)$; however, syntactically it is a sentence of the language of $T_{b n d}(\Pi)$. That is, $T_{\text {annot }}(\Pi, \rightsquigarrow)$ adds information to $T_{b n d}(\Pi)$, because $T_{\text {annot }}(\Pi, \rightsquigarrow)$ 's models are only the compatible bundles. The theorem is the following.

Theorem 4 (Inferred Extend Strand).

$$
\begin{aligned}
& \forall z_{0}, z_{1}: \mathrm{Z}, t, t_{0}, t_{1}: \top, m_{0}, m_{1}: \mathrm{M}, e s k_{0}, e s k_{1}, k_{0}, k_{1}: \mathrm{A} . \\
& \operatorname{htin}\left(z_{0}, 2 \text {, extend }\left(e s k_{0}, k_{0}, \operatorname{pcr}\left(m_{0}\right), t_{0}\right)\right) \\
& \wedge \operatorname{htin}\left(z_{1}, 2, \operatorname{extend}\left(\operatorname{esk}_{1}, k_{1}, \operatorname{pcr}\left(m_{1}\right), t_{1}\right)\right) \\
& \wedge\left(z_{0}, 1\right) \ll\left(z_{1}, 0\right) \wedge m_{1} \text { has } t \\
& \supset \operatorname{subterm}\left(\operatorname{ex}\left(t_{0}, m_{0}\right), m_{1}\right) \\
& \vee \exists z: \mathrm{Z}, m: \mathrm{M}, \text { esk, } k: \mathrm{A} \text {. } \\
& \operatorname{htin}(z, 2, \text { extend }(\text { esk, } k, p c r(m), t)) \\
& \wedge\left(z_{0}, 1\right) \ll(z, 0) \wedge(z, 1) \ll\left(z_{1}, 0\right)
\end{aligned}
$$

Theorem 4 implies that Fig. 6 has an additional extend strand, as shown in Fig. 8. Restarting CPSA with $\mathbb{A}_{0}$ enriched with all of this additional information, we learn that no such execution is possible. This justifies Security Goal 1. 


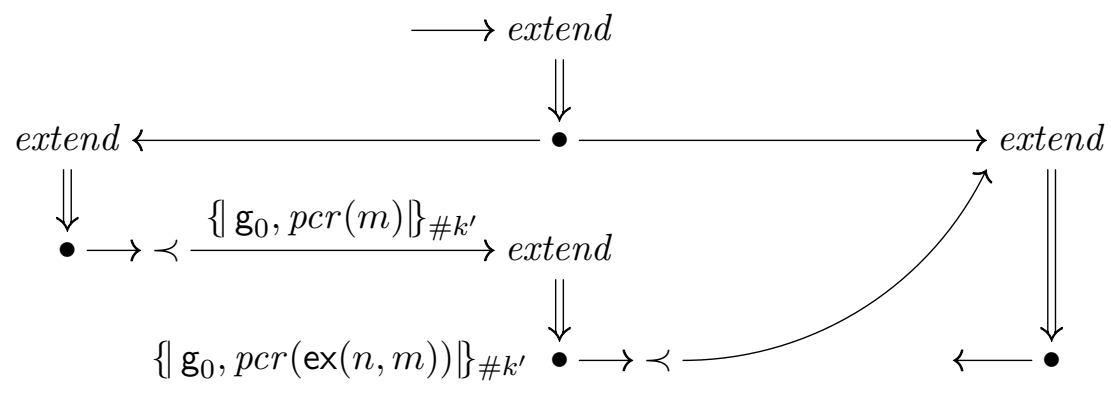

Figure 8: Inferred Extend Strand

Our Method. We have now completed an illustration of the hybrid method for analyzing a protocol with state. We took the following key steps.

1. We defined states and a transition relation representing a TPM fragment. We proved a key lemma (Lemma 1) in the resulting theory $T_{\text {state }}(\rightsquigarrow)$.

2. We defined the envelope protocol as a PVs theory $T_{b n d}(\Pi)$. We encoded the states as certain encrypted messages, and used state-passing to represent the actions of the TPM in protocol roles. The encoding function is an injective function $g$. We connect $\cdots-t_{0} \Rightarrow+t_{1} \cdots$, as a state-passing representation, with $T_{\text {state }}(\rightsquigarrow)$ by annotating the role with the annotation:

$$
\left\{\left(m_{0}, m_{1}\right) \mid t_{0}=g\left(m_{0}\right) \wedge t_{1}=g\left(m_{1}\right)\right\} \cap \rightsquigarrow .
$$

We prove bridge lemmas along the lines of Lemma 3.

3. Independently, we define $\Pi$ in the CPSA input language, and query CPSA with a starting point $\mathbb{A}_{0}$ as in our security goal. We translate the results in the form of state analysis sentences such as Lemma 2, which we use within PVS as axioms.

4. From a state analysis sentence and bridge lemmas, we deduce conclusions about all compatible bundles of $\Pi$ and $\rightsquigarrow$. Thm. 4 was an example. These theorems may already establish our security goals.

5. Alternatively, the conclusions about compatible bundles may give us an enriched starting point, which we can bring back into CPSA, as we 
did here to determine that Security Goal 1 is achieved, and $\mathbb{A}_{0}$ cannot appear in any compatible bundle.

We have also applied this method to several simple protocols besides the Envelope Protocol. The steps in applying the method are always the same. While the application of these ideas is routine, it is quite time consuming. A goal of future research is to automate much more of the method.

But why annotate events with subsets of the transition relation rather than elements of it? The extend role does not guarantee it receives a statebearing message of the form $\left\{\left|\mathrm{g}_{0}, \operatorname{pcr}\left(m_{0}\right)\right|\right\}_{\# k}$. It says only that the incoming message has the form $\left\{\left|\mathrm{g}_{0}, t_{0}\right|\right\}_{\# k}$. We must eliminate strands in which $t_{0}$ is not in the range of the pcr function. That is why we use the annotation shown in Step 2.

A bundle in which a received state encoding message is not in the range of the pcr function will have a node annotated with the empty set. This bundle does not respect state semantics and is eliminated from consideration by the definition of compatibility.

\section{Related Work and Conclusion}

Related Work. The problem of reasoning about protocols and state has been an increasing focus over the past several years. Protocols using Trusted Platform Modules (TPMs) and other hardware security modules (HSMs) have provided one of the main motivations for this line of work.

A line of work was motivated by HSMs used in the banking industry [16, 26]. This work identified the effects of persistent storage as complicating the security analysis of the devices. Much work explored the significance of this problem in the case of PKCS \#11 style devices for key management [5, 6, 12]. These papers, while very informative, exploited specific characteristics of the HSM problem; in particular, the most important mutable state concerns the attributes that determine the usage permitted for keys. These attributes should usually be handled in a monotonic way, so that once an attribute has been set, it will not be removed. This justifies using abstractions that are more typical of standard protocol analysis.

In the TPM-oriented line of work, an early example using an automatabased model was by Gürgens et al. [13]. It identified some protocol failures due to the weak binding between a TPM-resident key and an individual

person. Datta et al.'s "A Logic of Secure Systems" [9] presents a dynamic 
logic in the style of PCL [8] that can be used to reason about programs that both manipulate memory and also transmit and receive cryptographically constructed messages. Because it has a very detailed model of execution, it appears to require a level of effort similar to (multithreaded) program verification, unlike the less demanding forms of protocol analysis.

Mödersheim's set-membership abstraction [20] works by identifying all data values (e.g. keys) that have the same properties; a change in properties for a given key $K$ is represented by translating all facts true for $K$ 's old abstraction into new facts true of $K$ 's new abstraction. The reasoning is still based on monotonic methods (namely Horn clauses). Thus, it seems not to be a strategy for reasoning about TPM usage, for instance in the envelope protocol.

The paper [14] by one of us developed a theory for protocols (within strand spaces) as constrained by state transitions, and applied that theory to a fair exchange protocol. It introduced the key notion of compatibility between a protocol execution ("bundle") and a state history. In the current paper we will also rely on the same notion of compatibility, which was somewhat hidden in [14]. However, the current paper does not separate the protocol behavior from state history as sharply as did [14].

A group of papers by Ryan with Delaune, Kremer, and Steel [10, 11], and with Arapinis and Ritter [3] aim broadly to adapt ProVerif for protocols that interact with long-term state. ProVerif $[4,1]$ is a Horn-clause based protocol analyzer with a monotonic method: in its normal mode of usage, it tracks the messages that the adversary can obtain, and assumes that these will always remain available. Ryan et al. address the inherent non-monotonicity of adversary's capabilities by using a two-place predicate att $(u, m)$ meaning that the adversary may possess $m$ at some time when the long-term state is $u$. In [3], the authors provide a compiler from a process algebra with state-manipulating operators to sets of Horn clauses using this primitive. In [11], the authors analyze protocols with specific syntactic properties that help ensure termination of the analysis. In particular, they bound the state values that may be stored in the TPMs. In this way, the authors verify two protocols using the TPM, including the envelope protocol.

One advantage of the current approach relative to the ProVerif approach is that it works within a single comprehensive framework, namely that of strand spaces. Proofs about state within PVs succeeded only when definitions and lemmas were properly refined, and all essential details represented. As a result, our confidence is high that our proofs about protocols have their 
intended meaning.

Conclusion. The proof of the Envelope Protocol security goal presented here shows a detailed example of our method for applying CPSA to systems that include a state component. CPSA was coupled with about 2400 lines of PVS specifications to produce a proof of a difficult security goal. The method is sound due to the use of the common foundation of strand space theory for all reasoning.

The approach could be improved in two main ways. First, the proofs within PVS are strenuous. We would like to develop a method in whichapart perhaps from a few key reusable lemmas in the state theory $T_{\text {state }}(\rightsquigarrow)$ the remainder of the reasoning concerning both state and protocol behavior occurs automatically in CPSA's automated, enrich-by-need manner. Second, there is some artificiality in the state-threading representation that we have used here. It requires the protocol description to make explicit the details of the full state, and to express each state change in a syntactic, templatebased form. Moreover, the state information is also redundantly encoded in the annotations that appear in $T_{\text {annot }}(\Pi, \rightsquigarrow)$. Our earlier work [14] instead encapsulated all of the state information in a labeled transition relation. The protocol definitions contain only a type of "neutral node" which are neither transmissions nor receptions. These nodes are associated with the same labels as appear in labeled transitions. This allows us to define "compatibility," and to work with protocol and state definitions as independent modules. We intend also to explore this style of definition.

Acknowledgment. We are grateful to Ed Zieglar for discussions and support.

\section{References}

[1] Martín Abadi and Bruno Blanchet. Analyzing security protocols with secrecy types and logic programs. Journal of the ACM, 52(1):102-146, January 2005.

[2] K. Ables and M. Ryan. Escrowed data and the digital envelope. Trust and Trustworthy Computing, pages 246-256, 2010. 
[3] Myrto Arapinis, Eike Ritter, and Mark Dermot Ryan. Statverif: Verification of stateful processes. In Computer Security Foundations Symposium (CSF), pages 33-47. IEEE, 2011.

[4] Bruno Blanchet. An efficient protocol verifier based on Prolog rules. In 14th Computer Security Foundations Workshop, pages 82-96. IEEE CS Press, June 2001.

[5] Véronique Cortier, Gavin Keighren, and Graham Steel. Automatic analysis of the security of xor-based key management schemes. In Tools and Algorithms for the Construction and Analysis of Systems, pages 538552. Springer, 2007.

[6] Véronique Cortier and Graham Steel. A generic security api for symmetric key management on cryptographic devices. In Computer SecurityESORICS 2009, pages 605-620. Springer, 2009.

[7] Cas Cremers and Sjouke Mauw. Operational semantics and verification of security protocols. Springer, 2012.

[8] Anupam Datta, Ante Derek, John C. Mitchell, and Dusko Pavlovic. A derivation system and compositional logic for security protocols. Journal of Computer Security, 13(3):423-482, 2005.

[9] Anupam Datta, Jason Franklin, Deepak Garg, and Dilsun Kaynar. A logic of secure systems and its application to trusted computing. In Security and Privacy, 2009 30th IEEE Symposium on, pages 221-236. IEEE, 2009.

[10] Stéphanie Delaune, Steve Kremer, Mark D Ryan, and Graham Steel. A formal analysis of authentication in the TPM. In Formal Aspects of Security and Trust, pages 111-125. Springer, 2011.

[11] Stéphanie Delaune, Steve Kremer, Mark D. Ryan, and Graham Steel. Formal analysis of protocols based on TPM state registers. In IEEE Symposium on Computer Security Foundations. IEEE CS Press, June 2011.

[12] Sibylle Fröschle and Nils Sommer. Reasoning with past to prove PKCS\# 11 keys secure. In Formal Aspects of Security and Trust, pages 96-110. Springer, 2011. 
[13] Sigrid Gürgens, Carsten Rudolph, Dirk Scheuermann, Marion Atts, and Rainer Plaga. Security evaluation of scenarios based on the TCG's TPM specification. In Computer Security-ESORICS 200\%, pages 438-453. Springer, 2007.

[14] Joshua D. Guttman. State and progress in strand spaces: Proving fair exchange. Journal of Automated Reasoning, 48(2):159-195, 2012.

[15] Joshua D. Guttman. Establishing and preserving protocol security goals. Journal of Computer Security, 2014.

[16] Jonathan Herzog. Applying protocol analysis to security device interfaces. IEEE Security \& Privacy, 4(4):84-87, 2006.

[17] Bart Jacobs and Ichiro Hasuo. Semantics and logic for security protocols. Journal of Computer Security, 17(6):909-944, 2009.

[18] Moses D. Liskov, Paul D. Rowe, and F. Javier Thayer. Completeness of CPSA. Technical Report MTR110479, The MITRE Corporation, March 2011. http://www.mitre.org/publications/technical-papers/completeness-of-cpsa.

[19] Simon Meier, Cas Cremers, and David Basin. Efficient construction of machine-checked symbolic protocol security proofs. Journal of Computer Security, 21(1):41-87, 2013.

[20] Sebastian Mödersheim. Abstraction by set-membership: verifying security protocols and web services with databases. ACM Conference on Computer and Communications Security, pages 351-360, 2010.

[21] S. Owre, J. M. Rushby, , and N. Shankar. PVS: A prototype verification system. In Deepak Kapur, editor, 11th International Conference on Automated Deduction (CADE), volume 607 of Lecture Notes in Artificial Intelligence, pages 748-752, Saratoga, NY, jun 1992. Springer-Verlag. http://pvs.csl.sri.com.

[22] John D. Ramsdell. Deducing security goals from shape analysis sentences. The MITRE Corporation, April 2012. http://arxiv.org/abs/1204.0480. 
[23] John D. Ramsdell. Proving security goals with shape analysis sentences. Technical Report MTR130488, The MITRE Corporation, September 2013. http://arxiv.org/abs/1403.3563.

[24] John D. Ramsdell and Joshua D. Guttman. CPSA: A cryptographic protocol shapes analyzer, 2009. http://hackage. haskell .org/package/cpsa.

[25] F. Javier Thayer, Jonathan C. Herzog, and Joshua D. Guttman. Strand spaces: Proving security protocols correct. Journal of Computer Security, 7(2/3):191-230, 1999.

[26] Paul Youn, Ben Adida, Mike Bond, Jolyon Clulow, Jonathan Herzog, Amerson Lin, Ronald Rivest, and Ross Anderson. Robbing the bank with a theorem prover. In Security Protocols Workshop, 2007. Available at http://www.cl.cam.ac.uk/techreports/UCAM-CL-TR-644.pdf. 